\title{
Predictive analysis of superficial fluorescence patterns in non-melanoma skin cancer during photodynamic therapy
}

\section{Análisis predictivo de los patrones de fluorescencia superficiales en cáncer de piel no melanoma durante la terapia fotodinámica}

\author{
I. Salas-García*, F. Fanjul-Vélez, J. L. Arce-Diego ${ }^{\mathrm{S}}$ \\ Grupo de Técnicas Ópticas Aplicadas. Departamento TEISA, Universidad de Cantabria, Av. de los Castros S/N, \\ 39005 Santander, Cantabria, España.
}

(*) E-mail: isalas@teisa.unican.es

S: SEDOPTICA member

\author{
Received: 09/09/2015 Accepted: 16/02/2016 \\ DOI: $10.7149 /$ OPA.49.1.7
}

\begin{abstract}
:
Non-invasive treatment monitoring is one of the key elements to assess the photosensitizer activation during the photochemical process underlying Photodynamic Therapy (TFD) by its fluorescence. Furthermore the photosensitizer fluorescence can be employed to discriminate the pathological tissue from the healthy one. In this work the superficial fluorescence patterns in three different types of nonmelanoma skin cancer tumors and their photodynamic treatment response are analysed by a fluorescence based dosimetric model for PDT with topical MAL-PpIX. Results show differences of even more than $50 \%$ in the fluorescence patterns as the treatment progresses depending on the malignant tissue type. They demonstrate the great relevance of the biological media as an additional dosimetric factor within the photodynamic context. These results contribute to the development of a future customized therapy with the assistance of dosimetric tools to interpret the fluorescence images obtained during the treatment monitoring as well as in differential photodiagnosis.
\end{abstract}

Key words: Photodynamic Therapy, fluorescence, photodiagnosis, nonmelanoma skin cancer, dosimetry, photosensitizer.

\section{RESUMEN:}

La monitorización no invasiva es uno de los elementos claves para analizar la activación del fotosensibilizador durante el proceso fotoquímico subyacente a la Terapia Fotodinámica (TFD) mediante su fluorescencia. Esta última se puede emplear también para discriminar el tejido patológico del sano. En este trabajo se analizan los patrones de fluorescencia superficial en tres tipos diferentes de cáncer de piel no melanoma, así como su respuesta al tratamiento fotodinámico, mediante un modelo dosimétrico para TFD con MAL-PpIX. Los resultados obtenidos muestran diferencias incluso mayores del $50 \%$ en los patrones de fluorescencia a medida que progresa el tratamiento dependiendo del tipo de tejido maligno. Lo que demuestra la gran relevancia del medio biológico como factor dosimétrico adicional en el contexto fotodinámico. Estos resultados contribuyen al futuro de desarrollo de una terapia personalizada asistida mediante herramientas dosimétricas para interpretar las imágenes de fluorescencia obtenidas durante la monitorización del tratamiento y el fotodiagnóstico diferencial.

Palabras clave: Terapia Fotodinámica, fluorescencia, fotodiagnóstico, cáncer de piel no melanoma, dosimetría, fotosensibilizador 


\section{REFERENCIAS Y ENLACES}

[1] M. R. Hamblin, P. Mróz, Advances in Photodynamic Therapy: Basic, Translational and Clinical. Engineering in medicine \& Biology (2008).

[2] S. Choudhary, J. Tang, M. L. Elsaie, K. Nouri, "Lasers in the Treatment of Nonmelanoma Skin Cancer," Dermatol Surg 37, 409-425 (2011).

http://dx.doi.org/10.1111/j.1524-4725.2011.01928.x

[3] A. E. O'Connor, W. M. Gallagher A. T. Byrne, "Porphyrin and Nonporphyrin Photosensitizers in Oncology: Preclinical and Clinical Advances in Photodynamic Therapy," J. Photochem Photobiol BBiol 85, 1053-1074 (2009). http://dx.doi.org/10.1111/j.1751-1097.2009.00585.x

[4] B. C. Wilson, M. S. Patterson, L. Lilge, "Implicit and Explicit Dosimetry in Photodynamic Therapy: a New Paradigm," Lasers Med Sci 12, 182-199 (1997). http://dx.doi.org/10.1007/BF02765099

[5] B. Liu, T. J. Farrell, M. S. Patterson, "Comparison of noninvasive photodynamic therapy dosimetry methods using a dynamic model of ALA-PDT of human skin," Phys Med Biol 57, 825-841 (2012). http://dx.doi.org/10.1088/0031-9155/57/3/825

[6] M. A. Weston, M. S. Patterson, "Monitoring oxygen concentration during photodynamic therapy using prompt photosensitizes fluorescence," Phys Med Biol 58, 7039-7059 (2013). http://dx.doi.org/10.1088/0031-9155/58/20/7039

[7] R. M. Valentine, C. T. A. Brown, H. Moseley, S. Ibbotson, K. Wood, "Monte Carlo modeling of in vivo protoporphyrin IX fluorescence and singlet oxygen production during photodynamic therapy for patients presenting with superficial basal cell carcinomas," J Biomed Opt 16, 0480021-11 (2011). http://dx.doi.org/10.1117/1.3562540

[8] I. Salas-García, F. Fanjul-Vélez, J. L. Arce-Diego, "Superficial radially-resolved fluorescence and threedimensional photochemical time-dependent model for Photodynamic Therapy," Opt Lett 39, 18451848 (2014).

http://dx.doi.org/10.1364/OL.39.001845

[9] L. O. Svaasand, P. Wyss, M. T. Wyss, Y. Tadir, B. J. Tromberg, M. W. Berns, "Dosimetry model for photodynamic therapy with topically administered photosensitizers," Lasers in Surgery and Medicine 18, 139-149 (1996). http://dx.doi.org/10.1002/(SICI)1096-9101(1996)18:2<139::AID-LSM3>3.0.C0;2-T

[10] T. H. Foster, R. S. Murant, R. G. Bryant, R. S. Knox, S. L. Gibson, R. Hilf, “Oxygen consumption and diffusion effects in PDT," Radiation Research 126, 296-303 (1991). http://dx.doi.org/10.2307/3577919

[11] X. H. Hu, Y. Feng, J. Q. Lu, R. R. Allison, R. E. Cuenca, G. H. Downie, C. H. Sibata, "Modeling of a type II photofrin-mediated PDT process in a heterogeneous tissue phantom," Photochemistry and Photobiology 81, 1460-1468 (2005).

http://dx.doi.org/10.1562/2005-05-04-RA-513

[12] L. Wang, S. L. Jacques, L. Zheng, "MCML - Monte Carlo modeling of light transport in multi-layered tissues," Comput Meth Programs Biomed 47, 131-146 (1995). http://dx.doi.org/10.1016/0169-2607(95)01640-F

[13] I. Salas-García, F. Fanjul-Vélez, J. L. Arce-Diego, "Spatial photosensitizer fluorescence emission predictive analysis for photodynamic therapy monitoring applied to a skin disease," Opt Commun 285, 1581-1588 (2012). http://dx.doi.org/10.1016/j.optcom.2011.10.056

[14] S. L. Jacques, Monte Carlo Simulations of Fluorescence in Turbid Media, Handbook of Biomedical Fluorescence. B. W. Rogue and M. A. Mycek, eds. CRC Press (2003).

[15] E. Salomatina, B. Jiang, J. Novak, A. N. Yaroslavsky, "Optical properties of normal and cancerous human skin in the visible and near-infrared spectral range," J Biomed Opt 11, 0640261-640269 (2006). http://dx.doi.org/10.1117/1.2398928

[16] I. Salas-García, F. Fanjul-Vélez, J. L. Arce-Diego, "Photosensitizer absorption coefficient modeling and necrosis prediction during photodynamic therapy," J Photochem Photobiol B-Biol 114, 79-86 (2012). http://dx.doi.org/10.1016/i.jphotobiol.2012.05.013 


\section{Introducción}

La Terapia Fotodinámica (TFD) consiste en la administración sistémica o tópica de un fotosensibilizador y posterior aplicación de la radiación óptica tras un periodo de incubación en el que el fotosensibilizador se acumula de forma selectiva en el tejido tumoral. Como resultado se producen agentes citotóxicos en presencia del oxígeno que dan lugar a la destrucción del tejido maligno [1]. Su naturaleza mínimamente invasiva y el uso de radiación no ionizante, ha extendido su aplicación en diversos ámbitos clínicos para el tratamiento del cáncer y otros tipos de lesiones. Especialmente como técnica de tratamiento en dermatología, donde se aplica para el tratamiento del cáncer de piel no melanoma y patologías precursoras. La incidencia de estas últimas ha aumentado significativamente en las últimas décadas debido en gran medida al aumento de la exposición a la radiación ultravioleta impuesta por las tendencias estéticas actuales [2]. Sin embargo, a pesar de los buenos resultados terapéuticos, persiste la necesidad de desarrollar una dosimetría de tratamiento personalizada para evitar una eliminación incompleta del tejido tumoral y posibles recidivas. Uno de los fotosensibilizadores más empleados en este ámbito es el Aminolevulinato de Metilo (MAL, Metvix ${ }^{\circledR}$ ). Un precursor del componente fotoactivo Protoporfirina IX (PpIX) que se acumula selectivamente en las células tumorales [3]. Al igual que otros fotosensibilizadores de la familia de las porfirinas, el protocolo clínico para la aplicación de TFD-Metvix ${ }^{\circledR}$ es fijo e independiente del tipo de paciente o lesión, aunque los resultados del tratamiento varían en función de ambos. Por lo que el desarrollo de dosimetría personalizada para TFD es necesario para optimizar la respuesta del paciente al tratamiento y evitar una posible persistencia tumoral o futura recidiva como consecuencia de una elección inadecuada de los parámetros del tratamiento. Para ello se han estudiado diferentes estrategias dosimétricas y factores que afectan al proceso fotodinámico mediante modelos predictivos [4]. Estos últimos han de tener en cuenta una gran cantidad de factores dinámicos y procesos interrelacionados que pueden limitar el adecuado transcurso del tratamiento. Lo que les confiere una capacidad muy valiosa para estimar a priori la respuesta del tejido bajo unas determinadas condiciones dosimétricas. Por lo que el uso de modelos predictivos presenta un gran interés para realizar una planificación personalizada del tratamiento que maximice su efectividad terapéutica. Sin embargo, a pesar del gran esfuerzo realizado en los últimos años, el desarrollo de un modelo completo para TFD que considere los complejos fenómenos inherentes al proceso fotodinámico permanece sin resolverse. La propagación óptica en el tejido biológico, la distribución del fotosensibilizador y la interacción fotoquímica entre otros, son cruciales para evaluar la progresión del tratamiento y estimar el efecto del tratamiento bajo un determinado contexto clínico. Además, sería de gran interés poder analizar la señal de fluorescencia emitida por el fotosensibilizador, no sólo con fines diagnósticos aprovechando su acumulación selectiva en el tumor, sino también como indicador de su degeneración para monitorizar la evolución de la terapia. Los modelos propuestos recientemente incluyen aproximaciones para los fenómenos fotofísicos y fotoquímicos principales, así como para la fluorescencia emitida por la PpIX en TFD-ALA aplicada en dermatología. Igualmente se ha estudiado la relación entre la fluorescencia del fotosensibilizador y la distribución de oxígeno singlete [5]. En este caso el modelo empleado es unidimensional y se aplicó en piel sana, por lo que no se considera ni la dependencia radial del proceso fotodinámico ni la influencia de las propiedades ópticas del tejido patológico sobre la distribución de la radiación óptica. Este tipo de modelos se han empleado también para estimar la concentración de oxígeno local a partir de la fluorescencia del fotosensibilizador [6], aunque en este caso el análisis de los componentes moleculares involucrados en la interacción fotoquímica se obtuvo a partir de una simple función escalón para la tasa de fluencia. Por lo que no se considera adecuadamente ni la geometría ni las propiedades ópticas del medio biológico. Ambas consideraciones se han tratado mediante una aproximación de Monte Carlo [7], aunque otros aspectos como la distribución inhomogénea del fotosensibilizador, la degradación del oxígeno inducida por el proceso fotodinámico o la dependencia radial del oxígeno singlete fueron despreciados. Las limitaciones indicadas anteriormente limitan la evaluación cuantitativa del proceso fotodinámico en un escenario clínico real, así como un análisis minucioso de la fluorescencia medida en la superficie del tumor. Recientemente se propuso un modelo que permite obtener la evolución temporal de la fluorescencia superficial, y en función de la profundidad, junto con la concentración de oxígeno singlete generada durante el tratamiento [8]. Ambos juegan un papel fundamental como indicadores del progreso del tratamiento y en definitiva, del daño oxidativo. Sin embargo, el potencial de este modelo para la práctica clínica considerando diferentes tipos de cáncer de piel no ha sido estudiado en profundidad. Tal y como se indicó anteriormente los modelos anteriores hacen referencia a piel sana o a un tipo de tumores aislado. En este trabajo se presenta un análisis completo de la respuesta fotodinámica y la potencialidad de la monitorización de la fluorescencia del 
fotosensibilizador en varios tumores comunes de cáncer de piel no melanoma (carcinoma basocelular nodular (CBCN), carcinoma basocelular infiltrativo (CBCI) y carcinoma de células escamosas (CCE)). Se han escogido estas tres patologías, aunque para alguna de ellas la TFD no esté aún aprobada, debido a su tipología como cáncer de piel no melanoma, y no lesiones precursoras, de tal manera que su potencial de riesgo hace interesante plantearse el uso de la TFD. El análisis llevado a cabo permite evaluar la relevancia del tipo de tumor empleando un modelo tridimensional para TFD que proporciona la evolución de los principales fenómenos involucrados en el proceso fotodinámico junto con los patrones de fluorescencia superficiales y en profundidad a medida que progresa el tiempo de tratamiento. Los resultados específicos para cada tipo de tumor podrían ser útiles como herramienta de asistencia clínica tanto para planificar la respuesta al tratamiento como para monitorizar su progresión. En la sección 2 se describe brevemente el modelo dosimétrico para TFD-MAL empleado. Su aplicación en los tres tipos de cáncer de piel se presenta en la sección 3 junto a los resultados obtenidos. Finalmente en la sección 4 se presentan las conclusiones más relevantes de este trabajo.

\section{Descripción del modelo dosimétrico para la monitorización de fluorescencia superficial durante la TFD-MAL}

A continuación se describe brevemente la fundamentación del modelo empleado para obtener la evolución de los fenómenos involucrados en la TFD. Tal y como se indicó anteriormente, el fotosensibilizador de uso tópico MAL, es un precursor del elemento fotoactivo PpIX. Tras la administración tópica del MAL en la superficie cutánea, se produce un proceso de difusión a través de las diferentes capas de la piel y a continuación una transformación metabólica en la PpIX. Diferentes estudios han demostrado la gran influencia que tiene la capa córnea de la epidermis en el proceso de difusión del precursor hacia las capas más profundas de la piel y su distribución inhomogénea en el tejido. Para obtener dicha distribución durante el periodo de incubación de 3 horas impuesto por el protocolo para Metvix, se empleó la ley de difusión de Fick teniendo en cuenta la permeabilidad de la capa córnea $K$ y el coeficiente de difusión $D$ en el tejido tal y como se indica en (1) [9]. Donde $\tau$ es el tiempo de relajación del precursor como consecuencia de la generación del fotosensibilizador y otros procesos como el flujo linfático y la perfusión sanguínea y $M_{0}$ es la concentración inicial de precursor aplicada en la superficie del tumor. Esta última se obtuvo a partir de las indicaciones relacionadas con la administración de la crema Metvix ${ }^{\circledR}$ que se recogen en el protocolo clínico, considerando en todos los casos una lesión con una extensión superficial circular, la densidad de precursor en la crema y su masa molecular.

$$
M(t)=M_{o} \int_{0}^{t}\left(\frac{K}{\sqrt{D \pi t^{\prime}}} e^{-\frac{z^{2}}{4 D t^{\prime}}}-\frac{K^{2}}{D} e^{\frac{K}{D} z} e^{\frac{K^{2}}{D} t^{\prime}} \operatorname{erfc}\left(\frac{K}{\sqrt{D}} \sqrt{t^{\prime}}+\frac{z}{2 \sqrt{D t^{\prime}}}\right)\right) e^{-\frac{t^{\prime}}{\tau}} d t^{\prime}
$$

La concentración de PpIX producida endógenamente, $S_{0}$, es proporcional a la concentración instantánea de MAL, $M$, cuando el tiempo de relajación de la PpIX es mucho más pequeño que el tiempo de difusión del MAL y se puede obtener con la expresión (2).

$$
S_{0}(t)=\varepsilon_{p} \frac{\tau_{p}}{\tau_{a \rightarrow p}} M(t)
$$

La interacción fotoquímica que tiene lugar entre la luz, el fotosensibilizador y el oxígeno presente en el tejido se modeló mediante un sistema de ecuaciones diferenciales (3)-(8) que permite obtener la evolución temporal de los componentes moleculares en los diferentes puntos de la muestra de tejido [10, 11]. Las soluciones del sistema de ecuaciones se obtuvieron mediante una herramienta de resolución de ecuaciones diferenciales disponible en la plataforma Matlab ${ }^{\circledR}$. En estas ecuaciones, $\left[S_{0}\right]$ es la concentración de fotosensibilizador en estado base, $\left[S_{1}\right]$ es la concentración de fotosensibilizador en estado singlete excitado, $[T]$ es la concentración de fotosensibilizador en estado triplete excitado, $\left[{ }^{3} O_{2}\right]$ es la concentración de oxígeno en estado base, $\left[{ }^{1} O_{2}\right]$ es la concentración de oxígeno singlete, $[R]$ es la concentración de receptores intracelulares del oxígeno singlete, $[C]_{i}$ es la concentración se scavengers, $\tau_{1}$ es el tiempo de relajación del estado $S_{1}$ a $S_{0}, \tau_{3}$ es el tiempo de relajación del estado $T$ a $S_{0}$, $\tau_{0}$ es el tiempo de relajación del estado ${ }^{1} \mathrm{O}_{2}$ a ${ }^{3} \mathrm{O}_{2}, \eta_{10}$ es el rendimiento cuántico de transición del estado $S_{1}$ a $S_{0}$, 
$\eta_{13}$ es el rendimiento cuántico de transición de $S_{1}$ a $T, \eta_{30}$ es el rendimiento cuántico de transición de $T$ a $S_{0}, \eta_{0}$ es el rendimiento cuántico de transición de ${ }^{1} O_{2}$ a ${ }^{3} O_{2}, \alpha_{s}$ es el factor de eficiencia para la transferencia de energía de $T$ a ${ }^{3} \mathrm{O}_{2}, k p b$ es la tasa de fotoquemado biomolecular, $k c x$ es la tasa de citotoxicidad biomolecular, $k s c$ es la tasa de reacción del ${ }^{1} \mathrm{O}_{2}$ con varios scavengers, $v$ es la velocidad de la luz en el tejido, $\rho$ es la densidad de fotones, $\sigma_{p s a}$ es la sección transversal de absorción de las moléculas de $S_{0}, P$ es la tasa de difusión y perfusión de oxígeno y $U$ es la tasa de reparación de daño celular.

$$
\begin{gathered}
\frac{d\left[S_{0}\right]}{d t}=-v \rho \sigma_{p s a}\left[S_{0}\right]-k p b\left[{ }^{1} O_{2}\right]\left[S_{0}\right]+\frac{\eta_{10}}{\tau 1}\left[S_{1}\right]+\frac{\eta_{30}}{\tau 3}[T]+\frac{\alpha s}{\tau 3}[T]\left[{ }^{3} O_{2}\right] ; \\
\frac{d\left[S_{1}\right]}{d t}-\frac{1}{\tau 1}\left[S_{1}\right]+v \rho \sigma_{p s a}\left[S_{0}\right] \\
\frac{d[T]}{d t}=-\frac{\eta_{30}}{\tau 3}[T]-\frac{\alpha s}{\tau 3}[T]\left[{ }^{3} O_{2}\right]+\frac{\eta_{13}}{\tau 1}\left[S_{1}\right] ; \\
\frac{d\left[{ }^{3} O_{2}\right]}{d t}=-\frac{\alpha s}{\tau 3}[T]\left[{ }^{3} O_{2}\right]+\frac{\eta_{0}}{\tau 0}\left[{ }^{1} O_{2}\right]+P ; \\
\frac{d\left[{ }^{1} O_{2}\right]}{d t}=-k p b\left[S_{0}\right]\left[{ }^{1} O_{2}\right]-k c x[R]\left[{ }^{1} O_{2}\right]-k s c[C]\left[{ }^{1} O_{2}\right]-\frac{\eta_{0}}{\tau_{0}}\left[{ }^{1} O_{2}\right]+\frac{\alpha s}{\tau 3}[T]\left[{ }^{3} O_{2}\right] ; \\
\frac{d[R]}{d t}=-k c x\left[{ }^{1} O_{2}\right][R]+U
\end{gathered}
$$

La distribución de la radiación óptica de excitación en medios turbios, fuertes dispersores de la luz, y con grandes variaciones en sus propiedades ópticas, como son los tejidos biológicos, se puede obtener en una muestra de tejido en tres dimensiones mediante la Teoría de Transporte de la Radiación (RTT) asumiendo scattering múltiple y despreciando los efectos de interferencia y polarización. En el estado estacionario cuando la fuente de luz ha iluminado la muestra de tejido durante el tiempo suficiente de forma que los niveles de luz han alcanzado el equilibrio, y en una región libre de fuentes, la ecuación RT puede expresarse como en la ecuación (9), donde el parámetro básico es la intensidad específica $I(r, \hat{s})$ o potencia por unidad de área por unidad de ángulo sólido. Para resolver la ecuación RT en el estado estacionario se empleó el método de Monte Carlo de Wang y Jacques [12]. Esta implementación es multicapa, con sus bordes perpendiculares al haz de la fuente óptica y por lo tanto adecuada para el cálculo de la distribución óptica en medios estratificados como la piel. En este trabajo se consideraron las propiedades ópticas de cada capa de tejido a la longitud de onda de la TFD (635 nm).

$$
\hat{s} \cdot \bar{\nabla} I(r, \hat{s})=-\left(\mu_{a}+\mu_{s}\right) I(r, \hat{s})+\frac{\mu_{s}}{4 \pi} \int_{4 \pi} p\left(\hat{s} \cdot \hat{s}^{\prime}\right) I\left(r, \hat{s}^{\prime}\right) d \Omega^{\prime}
$$

La emisión de fluorescencia del fotosensibilizador en cada punto de la muestra del tejido (705 nm) se obtuvo a partir de la densidad de fotones de excitación absorbidos por las moléculas de fotosensibilizador y el rendimiento cuántico de fluorescencia $\eta_{10}$ [13]. La distribución espacial no uniforme de fluoróforo y su variación temporal provoca que la densidad de potencia generada en las fuentes emisoras de fluorescencia en cada posición del tejido varíen a lo largo del tratamiento como se expresa en (10). En esta ecuación $v$ es la velocidad de la luz en el tejido, $\rho$ es la densidad local de fotones y $\sigma_{p s a}$ es la sección transversal de absorción delas moléculas de fotosensibilizador. $E_{\text {foton } n_{\text {lem }}}=h \cdot c / \lambda_{e m}$ es la energía de fotón a la longitud de onda de emisión de fluorescencia $\lambda_{e m}, h$ es la constant de Planck y $c$ es la velocidad de la luz en el vacío.

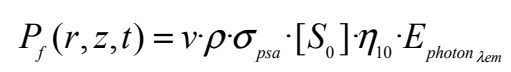

A partir de las fuentes de fluorescencia es posible obtener la fluorescencia debida a las moléculas de fotosensibilizador que escapa de la superficie del tumor. Para ello, se empleó otra aproximación del método de Monte Carlo [14], que fue modificada para considerar fuentes de fluorescencia locales que 
sufren una variación espacial y temporal dinámica [8]. Así, en un instante determinado del tratamiento, la fluorescencia total que escapa de la superficie del tumor $J_{f}$ en una determinada posición radial $r$ se puede obtener acumulando el flujo que escapa en superficie producido por cada fluente de potencia de fluorescencia tal y como se expresa en (11). En esta ecuación $r_{s} \mathrm{y} z_{s}$ son las coordenadas de la fuente de fluorescencia, $\Delta V\left(r_{s}, z_{s}\right)$ es el volumen incremental asociado con la posición de la fuente y $T\left(r_{s}, z_{s}, r\right)$ es la función de transferencia de fluorescencia desde la fuente hasta la superficie del tejido [8].

$$
J_{f}(r, t)=\sum_{r_{s}} \sum_{z_{s}} P_{f}\left(r_{s}, z_{s}, t\right) \Delta V\left(r_{s}, z_{s}\right) T\left(r_{s}, z_{s}, r\right)
$$

\section{Aplicación del modelo: resultados y discusión}

El modelo dosimétrico para TFD-MAL descrito anteriormente se aplicó a tres tipos diferentes de cáncer de piel no melanoma: CBCI, CBCN y CCE. Se consideró en todos los casos una lesión de radio superficial $1 \mathrm{~cm}$ y $3 \mathrm{~mm}$ de profundidad. Las propiedades ópticas empleadas para los diferentes tipos de tejido a la longitud de onda de tratamiento y de emisión de fluorescencia se recogen en la Tabla 1 [15]. En todos los casos se consideró un haz óptico cilíndrico de $0.3 \mathrm{~cm}$ de radio perpendicular a la muestra de tejido, una irradiancia de $100 \mathrm{~mW} / \mathrm{cm}^{2}$ y un tiempo de radiación de 10 minutos.

TABLA 1. Propiedades ópticas de los tejidos a las longitudes de onda de excitación y de emisión ( $\mu_{a}$ : coeficiente de absorción; $\mu_{s}$ : coeficiente de scattering).

\begin{tabular}{|c|c|c|}
\hline \multicolumn{3}{|c|}{ Longitud de onda de excitación $(635 \mathrm{~nm})$} \\
\hline \hline Tipo de tejido & $\mu_{a}\left(\mathrm{~cm}^{-1}\right)$ & $\mu_{s}\left(\mathrm{~cm}^{-1}\right)$ \\
\hline \hline CBCI & 1.5 & 142.85 \\
CBCN & 1.5 & 104.76 \\
CCE & 2 & 95.238 \\
\hline \hline \multicolumn{2}{|c|}{ Longitud de onda de emisión $(705 \mathrm{~nm})$} \\
\hline \hline Tipo de tejido & $\mu_{a}\left(\mathrm{~cm}^{-1}\right)$ & $\mu_{s}\left(\mathrm{~cm}^{-1}\right)$ \\
\hline \hline CBCI & 1.5 & 100 \\
\hline CBCN & 0.8 & 90 \\
\hline CCE & 1 & 85 \\
\hline \hline
\end{tabular}

La concentración inicial de MAL aplicada en la superficie de la lesión se calculó en base a las especificaciones del protocolo clínico para Metvix ${ }^{\circledR}$, donde se recomienda la aplicación de 1 mm de grosor de crema cubriendo $5 \mathrm{~mm}$ extra de piel sana alrededor de la patología y un periodo de incubación de 3 horas antes de aplicar la radiación óptica. Así, teniendo en cuenta una densidad de 160 mg de MAL por gramo de crema Metvix ${ }^{\circledR}$ y su masa molecular, se obtuvo una concentración inicial de precursor de PpIX de $4.5 \cdot 10^{20} \mathrm{~cm}^{-3}$ sobre la superficie del tumor. Se adoptaron valores empleados comúnmente tanto para el coeficiente de difusión a través de la epidermis y la dermis, $0.69 \cdot 10^{-10} \mathrm{~m}^{2} / \mathrm{s}$, así como para la permeabilidad de la capa cornea, $10^{-6} \mathrm{~m} / \mathrm{s}$. Una descripción más detallada de los parámetros empleados para el modelado de la interacción fotoquímica se puede consultar en trabajos previos [16].

En la Fig. 1 a) y b) se representan los patrones de absorción de fluorescencia en el CBCI tras 6 s y 600 s de irradiación. Los resultados obtenidos reflejan claramente no sólo la esperada disminución de la absorción de fluorescencia (nótese la diferente escala) a medida que progresa el tratamiento, sino también el ensanchamiento de las zonas de mayor absorción dentro de la muestra de tejido. Este último efecto es una consecuencia directa del incremento del camino que recorren los fotones de fluorescencia generados en las zonas más profundas, y en consecuencia del mayor número de eventos de scattering al que se ven sometidos. La expansión de la absorción de fluorescencia hacia las zonas más profundas del tejido se puede asociar claramente con una mayor producción de moléculas de oxígeno singlete en dichas zonas tal y como se puede observar en las Figs. 1 c) y d). Una mayor absorción de radiación óptica genera una mayor señal de fluorescencia y, a la vez, desencadena el proceso fotodinámico de generación de oxígeno singlete citotóxico. Lo que indica un retraso del daño oxidativo mediado por por el ${ }^{1} \mathrm{O}_{2}$ en esos puntos del tumor, lo que podría llevar a una persistencia tumoral en las zonas más profundas del tumor en caso de que el tiempo de irradiación no fuese el suficiente para desencadenar la interacción fotoquímica. En estos 
casos, la monitorización espacio-temporal de la señal de fluorescencia emitida por el fotosensibilizador podría ser un indicador adecuado para estimar de forma no invasiva la generación del agente citotóxico a partir de la señal de fluorescencia detectada en la superficie tumoral durante el tratamiento fotodinámico. En la Fig. 2 se representa la dependencia temporal de la fluorescencia media que escapa de la superficie del CBCI en función de la coordenada radial. Se llevaron a cabo un total de 10 iteraciones del modelo, de naturaleza pseudoaleatoria, para cada tipo de cáncer de piel, empleando valores promedio de propiedades ópticas y fotoquímicas, y su distribución particular de fuentes de fluorescencia en diferentes instantes temporales. Las barras de error representan la desviación estándar de los valores de irradiancia óptica de fluorescencia en cada punto de la dirección radial para el total de 10 iteraciones. Tal y como se puede observar el aumento de la degradación del fotosensibilizador como consecuencia del fotoquemado y la absorción de la señal de fluorescencia particular en cada patología produce una disminución de la fluorescencia que escapa en la superficie del tumor a medida que progresa el tiempo de tratamiento. Igualmente se puede observar el efecto del radio del haz óptico sobre el área superficial de máxima emisión. Lo que pone de manifiesto la necesidad de disponer de modelos tridimensionales para evaluar la influencia del perfil del haz óptico empleado sobre la predicción obtenida.
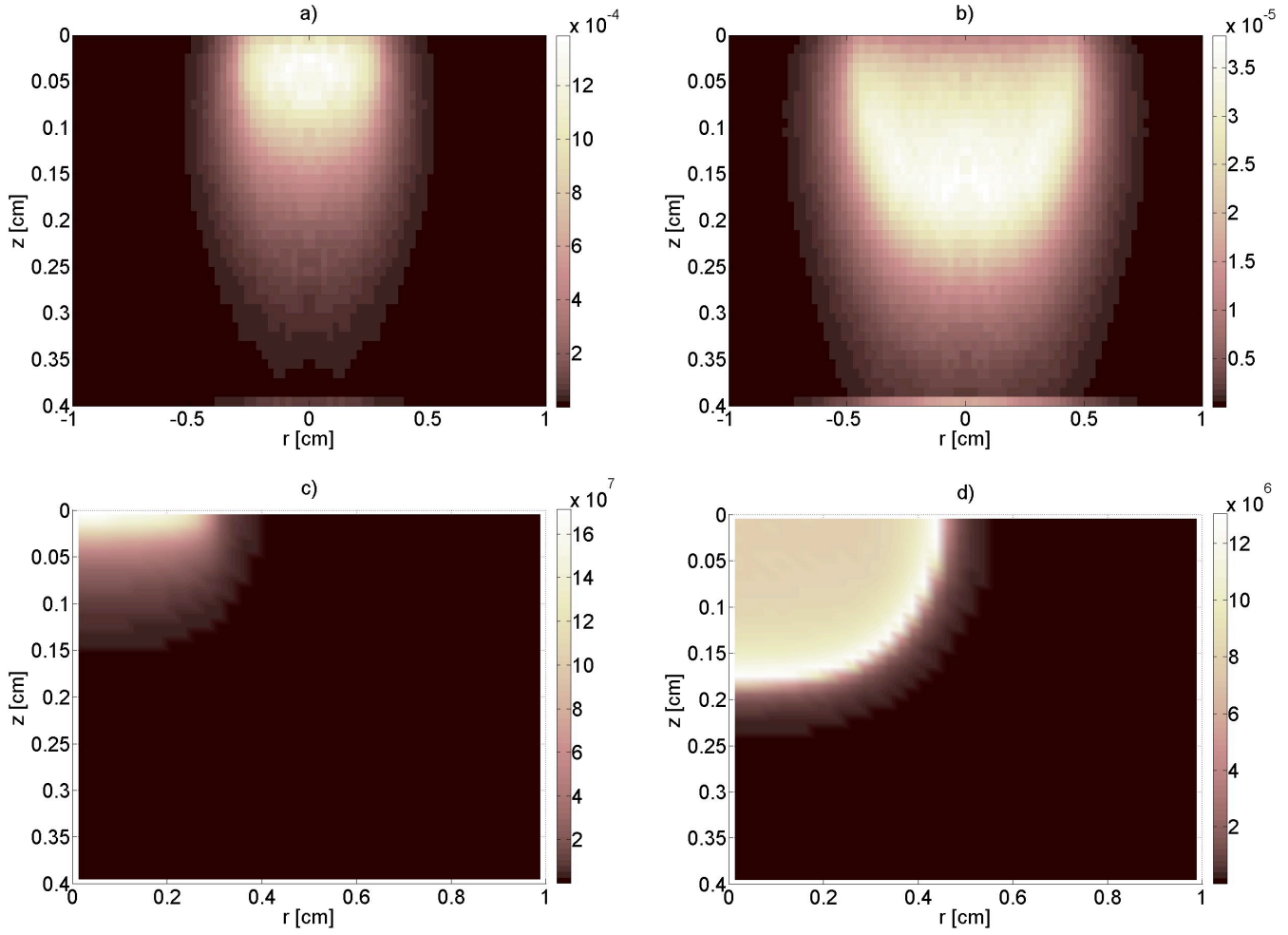

Fig.1. Absorción de fluorescencia (W / $\mathrm{cm}^{2}$ ) en el CBBI tras a) $6 \mathrm{~s}$ y b) $600 \mathrm{~s}$ de irradiación, y distribución de ${ }^{1} \mathrm{O}_{2}\left(\mathrm{~cm}^{-3}\right)$ en los mismos instantes temporales c) y d) respectivamente, (nótese la diferente escala en los gráficos).

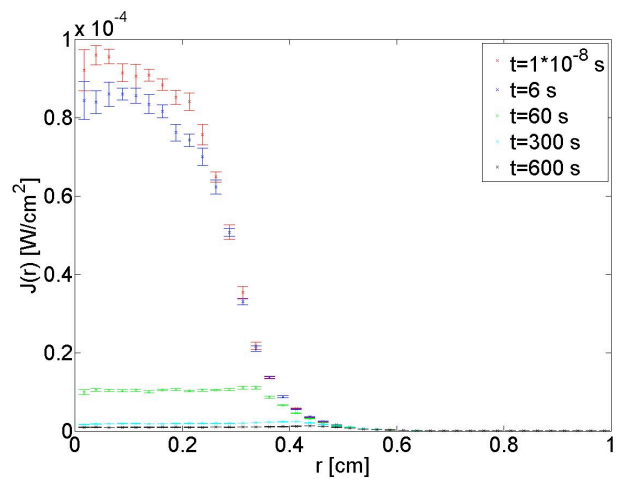

Fig.2. Fluorescencia que escapa en la superficie del CBCI $\left(\mathrm{W} / \mathrm{cm}^{2}\right)$ vs. componente radial en diferentes instantes temporales a lo largo del tratamiento. Las barras de error representan la desviación estándar de los valores de irradiancia óptica de fluorescencia en cada punto de la dirección radial para el total de 10 iteraciones. 
Los patrones de fluorescencia superficial obtenidos a medida que transcurre el tratamiento fotodinámico para los diferentes tipos de tejido maligno se representan en la Fig. 3 donde se pueden observar claras diferencias dependiendo del tejido tratado. Como se puede observar, a pesar de que su tendencia espaciotemporal es similar en los tres tipos de tumor (CBCI, CBCN, CCE) bajo las mismas condiciones de tratamiento, la fluorescencia detectada en la superficie varía para cada lesión en los diferentes instantes temporales a lo largo del tratamiento (instante temporal al comienzo del tratamiento Fig. 3 a), en el primer minuto Fig. 3 b), a mitad del tratamiento Fig. 3 c) y al final Fig. 3 d)).

La cuantificación estadística de las diferencias encontradas en el área de incidencia directa del haz óptico revela un incremento medio por encima del $50 \%$ entre el CBCN y el CBCI a lo largo de todo el tratamiento (50.39\% en el primer minuto, $58.22 \%$ a mitad del tratamiento y $56.51 \%$ al final). Esta diferencia se reduce ligeramente si se compara el CCE y el CBCI a $35.30 \%, 40.62 \%$ y $39.46 \%$ respectivamente. La discriminación entre patologías se reduce considerablemente entre el CBCN y el CCE. En cuyo caso la diferencia se reduce a $11.18 \%, 12.53 \%$ y $12.25 \%$. Este tipo de resultados son de gran ayuda para la monitorización personalizada de la evolución del tratamiento fotodinámico y además reflejan la importancia del tipo de tumor para planificar adecuadamente el tratamiento ya que los patrones de fluorescencia tienen una relación directa con la degradación del fotosensibilizador durante la reacción fotoquímica y en consecuencia con la generación de agente citotóxico. Igualmente las diferencias observadas en los instantes iniciales del tratamiento, a tan solo $1 \cdot 10^{-8} \mathrm{~s}$ cuando aún no se ha producido agente citotóxico, le confieren al modelo presentado utilidad desde el punto de vista del fotodiagnóstico diferencial. Las diferencias estadísticamente significativas en la emisión de fluorescencia superficial, así como la falta de solapamiento entre las desviaciones estándar, apoyan la viabilidad de herramientas predictivas tanto para la monitorización del tratamiento como para el fotodiagnóstico.
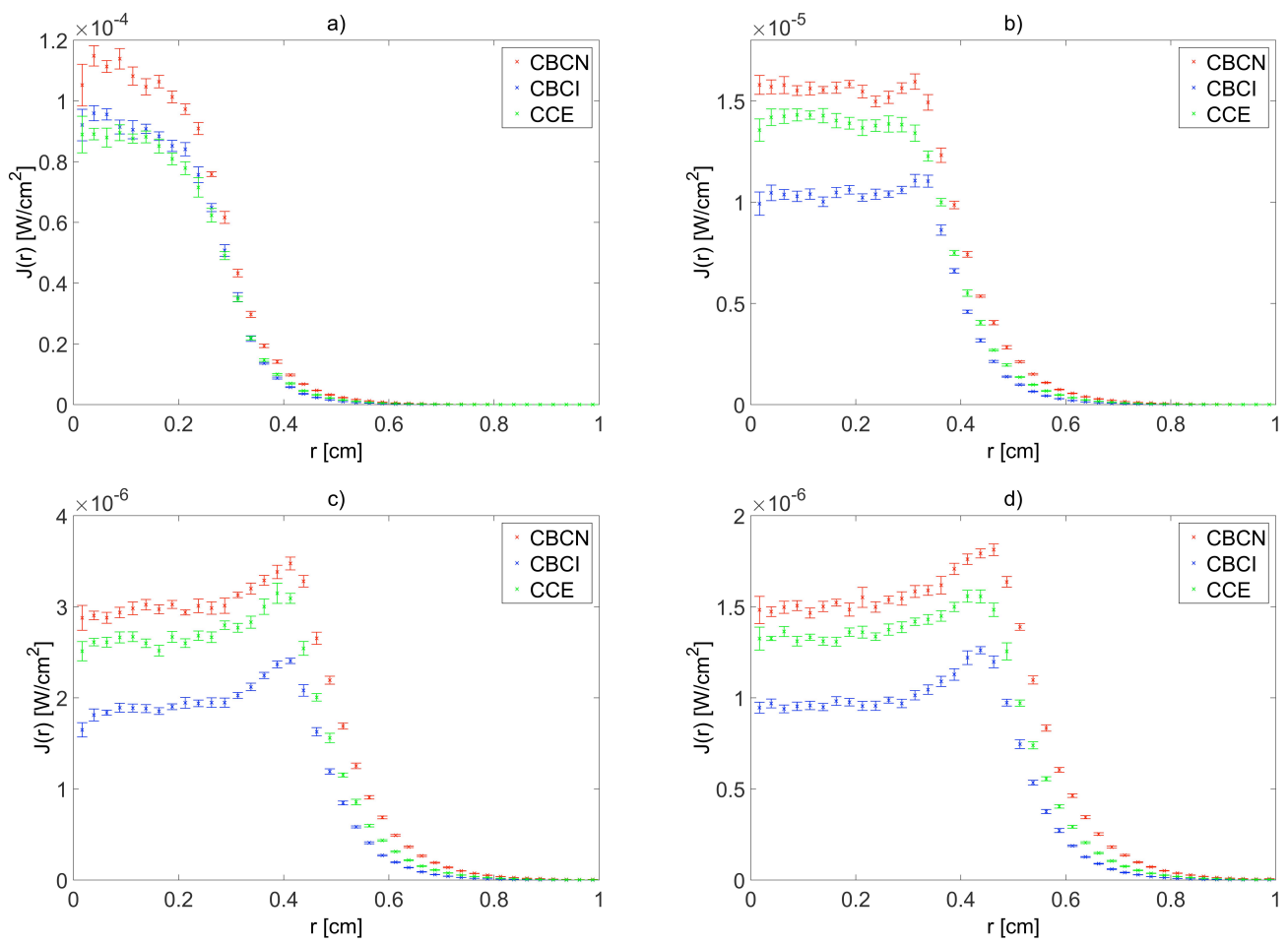

Fig.3. Fluorescencia que escapa en la superficie $\left(\mathrm{W} / \mathrm{cm}^{2}\right)$ del CBCN, CBCI y del CCE vs. la componente radial en diferentes instantes temporales del tratamiento: a) al comienzo $\left(1 \cdot 10^{-8} \mathrm{~s}\right)$, b) en el primer minuto, c) a la mitad y d) al final. Las barras de error representan la desviación estándar de los valores de irradiancia óptica de fluorescencia en cada punto de la dirección radial para el total de 10 iteraciones.

\section{Conclusiones}

Se ha analizado la fluorescencia superficial emitida por el fotosensibilizador MAL-PpIX durante el transcurso de la TFD en diferentes tipos de cáncer de piel no melanoma mediante un modelo predictivo. 
Este modelo predictivo contempla, por un lado, la distribución de la propagación óptica en el tejido biológico. Por otro, tiene en cuenta la distribución del fotosensibilizador tópico Metvix ${ }^{\circledR}$. Mediante un completo modelo fotoquímico se estima la distribución espacial de las fuentes de fluorescencia, y se genera la propagación de la radiación proveniente de dichas fuentes hasta la superficie del tejido.

Los resultados obtenidos revelan diferencias incluso superiores al 50 \% dependiendo del tipo de tumor y una relación inherente con la generación del agente citotóxico encargado de la destrucción de las células tumorales. De manera cuantitativa, estas diferencias se explicitan, entre el CBCN y el CBCI a lo largo de todo el tratamiento, en un $50.39 \%$ en el primer minuto, un $58.22 \%$ a mitad del tratamiento y un $56.51 \%$ al final. La diferencia se reduce ligeramente si se compara el CCE y el CBCI a $35.30 \%, 40.62 \%$ y $39.46 \%$ respectivamente. La discriminación entre patologías se reduce considerablemente entre el CBCN y el CCE. En cuyo caso la diferencia se reduce a $11.18 \%, 12.53 \%$ y $12.25 \%$. Como consecuencia, tanto la morfología como el tipo de tumor deben ser considerados como un factor dosimétrico clave para el desarrollo de herramientas predictivas que permitan una adecuada interpretación de la fluorescencia superficial emitida en tumores de cáncer de piel no melanoma fotosensibilizados mediante MAL-PpIX. Se plana extender este estudio a un mayor número de muestras y de patologías. Las aproximaciones dosimétricas basadas en fluorescencia necesitan de este tipo de análisis para asegurar la precisión de la monitorización. Lo que pone de manifiesto la aplicabilidad de este tipo de herramientas tanto en la planificación personalizada y monitorización del tratamiento fotodinámico, como en el fotodiagnóstico diferencial.

\section{Agradecimientos}

Este trabajo ha sido parcialmente financiado por el proyecto MAT2012-38664-C02-01, cofinanciado con fondos FEDER, y por la Fundación San Cándido. 